\title{
COSMOLOGY FROM GRAVITATIONAL LENS TIME DELAYS AND PLANCK DATA
}

\author{
S. H. Suyu ${ }^{1}$, T. Treu ${ }^{2,11}$, S. Hilbert ${ }^{3}$, A. Sonnenfeld ${ }^{2}$, M. W. Auger ${ }^{4}$, R. D. Blandford ${ }^{5}$, T. Collett ${ }^{4}$, F. Courbin $^{6}$,

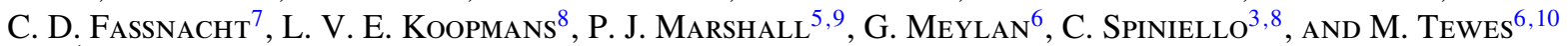 \\ ${ }^{1}$ Institute of Astronomy and Astrophysics, Academia Sinica, P.O. Box 23-141, Taipei 10617, Taiwan; suyu@ asiaa.sinica.edu.tw \\ ${ }^{2}$ Department of Physics, University of California, Santa Barbara, CA 93106, USA \\ ${ }^{3}$ Max-Planck-Institut für Astrophysik, Karl-Schwarzschild-Strasse 1, D-85748 Garching, Germany \\ ${ }^{4}$ Institute of Astronomy, University of Cambridge, Madingley Road, Cambridge CB3 OHA, UK \\ ${ }^{5}$ Kavli Institute for Particle Astrophysics and Cosmology, Stanford University, 452 Lomita Mall, Stanford, CA 94035, USA \\ ${ }^{6}$ Laboratoire d'Astrophysique, Ecole Polytechnique Fédérale de Lausanne (EPFL), Observatoire de Sauverny, 1290-CH Versoix, Switzerland \\ ${ }^{7}$ Department of Physics, University of California, Davis, CA 95616, USA \\ ${ }^{8}$ Kapteyn Astronomical Institute, University of Groningen, P.O. Box 800, 9700-AV Groningen, The Netherlands \\ ${ }^{9}$ Department of Physics, University of Oxford, Keble Road, Oxford OX1 3RH, UK \\ ${ }^{10}$ Argelander-Institut für Astronomie, Auf dem Hügel 71, D-53121 Bonn, Germany \\ Received 2014 May 21; accepted 2014 May 22; published 2014 June 5
}

\begin{abstract}
Under the assumption of a flat $\Lambda \mathrm{CDM}$ cosmology, recent data from the Planck satellite point toward a Hubble constant that is in tension with that measured by gravitational lens time delays and by the local distance ladder. Prosaically, this difference could arise from unknown systematic uncertainties in some of the measurements. More interestingly_if systematics were ruled out—resolving the tension would require a departure from the flat $\Lambda$ CDM cosmology, introducing, for example, a modest amount of spatial curvature, or a non-trivial dark energy equation of state. To begin to address these issues, we present an analysis of the gravitational lens RXJ1131-1231 that is improved in one particular regard: we examine the issue of systematic error introduced by an assumed lens model density profile. We use more flexible gravitational lens models with baryonic and dark matter components, and find that the exquisite Hubble Space Telescope image with thousands of intensity pixels in the Einstein ring and the stellar velocity dispersion of the lens contain sufficient information to constrain these more flexible models. The total uncertainty on the time-delay distance is $6.6 \%$ for a single system. We proceed to combine our improved time-delay distance measurement with the WMAP9 and Planck posteriors. In an open $\Lambda$ CDM model, the data for RXJ1131-1231 in combination with Planck favor a flat universe with $\Omega_{\mathrm{k}}=0.00_{-0.02}^{+0.01}(68 \%$ credible interval (CI)). In a flat $w$ CDM model, the combination of RXJ1131-1231 and Planck yields $w=-1.52_{-0.20}^{+0.19}$ (68\% CI).
\end{abstract}

Key words: distance scale - galaxies: individual (RXJ1131-1231) - gravitational lensing: strong methods: data analysis

Online-only material: color figures

\section{INTRODUCTION}

The last few years have been hailed as the era of precision cosmology. Many different methods now point to the so-called concordance cosmology, characterized by a virtually flat geometry in a universe dominated by dark matter and dark energy (e.g., Hinshaw et al. 2013; Planck Collaboration et al. 2013). With precisions on many parameters now reaching the few percent level, it is extremely valuable to compare and contrast different probes. A comparison between independent probes is a robust way to test the accuracy of the measurements. Furthermore, certified tension between independent probes' measurements would require the falsification of the simplest models and potentially the discovery of new physics (Suyu et al. 2012).

A classic example is the interpretation of the cosmic microwave background (CMB) data. The power spectrum of the $\mathrm{CMB}$ anisotropies delivers an enormous amount of information about the high-redshift universe, but it is not directly sensitive to lower-redshift phenomena. Thus, inferring $w$ or the Hubble constant $\left(H_{0}\right)$ from the CMB data typically requires strong assumptions about the cosmological model (e.g., flatness) or the combination with lower-redshift probes. This is well exemplified by the Planck analysis (Paper XVI; Planck Collaboration et al. 2013). Assuming $\Omega_{\mathrm{k}}=0$ and $w=-1$,

\footnotetext{
${ }^{11}$ Packard Research Fellow.
}

$H_{0}=67.3 \pm 1.2 \mathrm{~km} \mathrm{~s}^{-1} \mathrm{Mpc}^{-1}$, in tension with that measured by various lower-redshift methods (e.g., Riess et al. 2011; Freedman et al. 2012; Chávez et al. 2012; Suyu et al. 2013). If confirmed, this tension would imply that the simplest flat $\Lambda \mathrm{CDM}$ is falsified. Given the high stakes, it is crucial to re-examine the uncertainties of each method, eliminating unaccounted for systematics.

The aim of this Letter is two-fold. First, we present a re-analysis of the gravitational lens system RXJ1131-1231 (Figure 1) discovered by Sluse et al. (2003). Following the work of Schneider \& Sluse (2013, hereafter SS13), we consider composite mass models for the main deflector galaxy (Section 2). The composite models consist of stellar and dark matter components, and are thus more realistic and flexible than the power-law models considered in our original analysis (Suyu et al. 2013, hereafter SU13). We show that even with this broader class of lens models, our deep Hubble Space Telescope (HST) images of the Einstein ring together with the stellar velocity dispersion measurement of the lens allow us to constrain the time-delay distance $\left(D_{\Delta t} \propto H_{0}^{-1}\right)$, a combination of angular diameter distances.

Second, having shown that uncertainties in the mass model are not significantly larger than our previous estimate, we proceed in Section 4 to combine our $D_{\Delta t}$ measurement with the recent CMB results from the Wilkinson Microwave Anisotropy Probe 


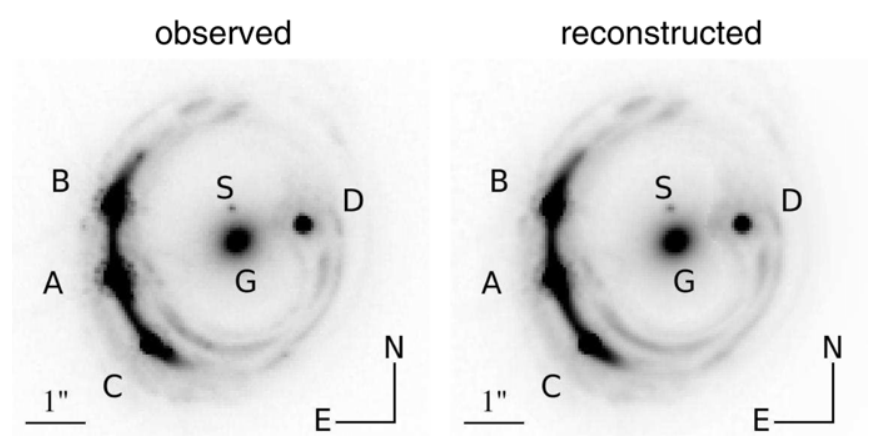

Figure 1. HST ACS image of RXJ1131-1231 in F814W filter. The background active galactic nucleus is lensed into four images (A, B, C, and D) by the primary lens galaxy $\mathrm{G}$ and its satellite S. Left: observed image. Right: reconstructed image based on the most probable composite model in Section 2.2.

9 year data (WMAP9; Hinshaw et al. 2013) and from Planck (Planck Collaboration et al. 2013). We conclude in Section 5.

Throughout this Letter, each quoted parameter estimate is the median of the marginalized posterior probability density function (PDF), with the uncertainties showing the 16th and 84th percentiles (i.e., the $68 \%$ credible interval $(\mathrm{CI})$ ).

\section{LENS MASS MODELS: POWER OF SPATIALLY EXTENDED EINSTEIN RINGS}

SU13 modeled the lens galaxy in RXJ1131-1231 with a power-law mass distribution that was motivated by several studies, including the X-ray observations of galaxies (Humphrey \& Buote 2010) and the Sloan Lens Advanced Camera for Surveys (SLACS; e.g., Koopmans et al. 2006, 2009; Gavazzi et al. 2007; Auger et al. 2010; Barnabè et al. 2011), which found that galaxies are well described by power-law mass distributions in regions covered by the data. Furthermore, the pixelated lens potential corrections applied by Suyu et al. (2009) to the gravitational lens B1608+656 was within $\sim 2 \%$ from a power law, validating the use of a simple power-law model. Here, we further assess the dependence of $D_{\Delta t}$ on the form of the mass model by employing two other forms that were considered by SS13: a cored power-law mass distribution, and a composite model of dark matter and baryons. In each case, we use the time delays from Tewes et al. (2013b) $)^{12}$ and the HST image (Figure 1; SU13) to constrain the lens model. The expressions for the likelihoods of the data are given in Section 6.2 of SU13.

\subsection{Cored Power-law Model}

The dimensionless surface mass density (convergence) of a cored elliptical power-law profile is given by

$$
\kappa_{\mathrm{cpl}}\left(\theta_{1}, \theta_{2}\right)=\frac{3-\gamma^{\prime}}{2}\left(\frac{\theta_{\mathrm{E}}}{\sqrt{q \theta_{1}^{2}+\theta_{2}^{2} / q+\theta_{\mathrm{c}}^{2}}}\right)^{\gamma^{\prime}-1},
$$

where $\left(\theta_{1}, \theta_{2}\right)$ are coordinates on the lens/image plane, $\gamma^{\prime}$ is the radial power-law slope (with $\gamma^{\prime}=2$ corresponding to isothermal), $\theta_{\mathrm{E}}$ is the Einstein radius, $q$ is the axis ratio, and $\theta_{\mathrm{c}}$ is the core radius. This is identical to the lens mass distribution in SU13 except for the non-zero $\theta_{c}$ here.

\footnotetext{
12 Based on monitorings of the COSmological MOnitoring of GRAvItational Lenses (COSMOGRAIL; e.g., Courbin et al. 2011; Tewes et al. 2013a) and Kochanek et al. (2006) teams.
}

Figure 1 shows a primary lens galaxy $\mathrm{G}$ and a satellite lens galaxy $\mathrm{S}$ that are surrounded by the Einstein ring of the lensed source. Following SU13 in the modeling procedure, we remodel the Advanced Camera for Surveys (ACS) image using the cored power-law profile for G. For simplicity, we fix the mass distribution of $\mathrm{S}$ to that of the most probable model in SU13 since the satellite impacts the $D_{\Delta t}^{\text {model }}$ measurement at the $<1 \%$ level. We also include an external shear contribution with strength $\gamma_{\text {ext }}$ and position angle $\phi_{\text {ext }}$. We use a grid of $50 \times 50$ intensity pixels on the source plane to model the spatially extended quasar host galaxy. These source pixels map to an annular region on the image plane containing the arcs that are visible in Figure 1. We sample the lens parameters and $D_{\Delta t}^{\text {model }}$ using the same Markov chain Monte Carlo (MCMC) methods as were used in SU13. The lensing data constrain the maximum $\theta_{\mathrm{c}}$ to be $0^{\prime} .005$ (95\% $\mathrm{CI}$ ), and $\theta_{\mathrm{c}}=0$ is compatible with the data. The marginalized values of the lens parameters, $D_{\Delta t}^{\text {model }}$, and the cosmological results are the same as those presented in SU13 within two significant digits.

\subsection{Composite Mass Model}

In the composite model, we treat baryons and dark matter individually. We model the baryonic mass distribution of the lens galaxy $\mathrm{G}$ as its observed light profile normalized by a constant $M / L$. The difference of two isothermal profiles mimics a Sérsic profile (Dutton et al. 2011) and provides efficient computation of lensing quantities:

$$
\begin{aligned}
L\left(\theta_{1}, \theta_{2}\right)= & \frac{L_{0}}{\left(1+q_{\mathrm{L}}\right)}\left[\frac{1}{\sqrt{\theta_{1}^{2}+\theta_{2}^{2} / q_{\mathrm{L}}^{2}+4 w_{\mathrm{c}}^{2} /\left(1+q_{\mathrm{L}}\right)^{2}}}\right. \\
& \left.-\frac{1}{\sqrt{\theta_{1}^{2}+\theta_{2}^{2} / q_{\mathrm{L}}^{2}+4 w_{\mathrm{t}}^{2} /\left(1+q_{\mathrm{L}}\right)^{2}}}\right],
\end{aligned}
$$

where $q_{\mathrm{L}}$ is the axis ratio, and $w_{c}$ and $w_{t}$ are profile parameters with $w_{\mathrm{t}}>w_{\mathrm{c}}$. We use two sets of the above profile with common centroid and position angle to fit the light distribution of G in the ACS image since a single one is inadequate (Claeskens et al. 2006; SU13). The optimized structural parameters are $\left(q_{\mathrm{L} 1}, w_{\mathrm{c} 1}, w_{\mathrm{t} 1}\right)=(0.88,2.0,2.5)$ and $\left(q_{\mathrm{L} 2}, w_{\mathrm{c} 2}, w_{\mathrm{t} 2}\right)=$ $(0.85,0.06,0.67)$, and are held fixed since the uncertainties on these parameters $(<2 \%)$ are negligible in terms of their effect on $D_{\Delta t}^{\text {model }}$ (SU13). For the dark matter halo, we adopt the standard Navarro-Frenk-White (NFW) profile (Navarro et al. 1996) whose three-dimensional density is

$$
\rho(r)=\frac{\rho_{0}}{\left(r / r_{\mathrm{s}}\right)\left(1+r / r_{\mathrm{s}}\right)^{2}},
$$

where $\rho_{0}$ is a normalization and $r_{\mathrm{s}}$ is the scale radius. We follow Golse \& Kneib (2002) for obtaining the deflection angles and lens potential of an elliptical NFW profile in projection. ${ }^{13}$ For the satellite, we model its mass distribution as a singular isothermal sphere centered on its light distribution. As in the previous cases, we allow for an external shear contribution.

We have 11 parameters in modeling the ACS image and time delays: a global $M / L$ of the baryons, the NFW parameters (centroid $\left(\theta_{1 \mathrm{~h}}, \theta_{2 \mathrm{~h}}\right)$, axis ratio $q_{\mathrm{h}}$, position angle $\phi_{\mathrm{h}}$, normalization $\kappa_{0, \mathrm{~h}}$, scale radius $r_{\mathrm{s}}$ ), satellite Einstein radius $\theta_{\mathrm{E}, \mathrm{S}}$, external shear $\gamma_{\mathrm{ext}}$, and $\phi_{\mathrm{ext}}$, and the modeled time-delay distance $D_{\Delta t}^{\text {model }}$.

\footnotetext{
13 Golse \& Kneib (2002) introduced the ellipticity into the lens potential, and Sand et al. (2008) showed that this yields valid elliptical surface mass density when $q_{\mathrm{h}} \gtrsim 0.8$.
} 


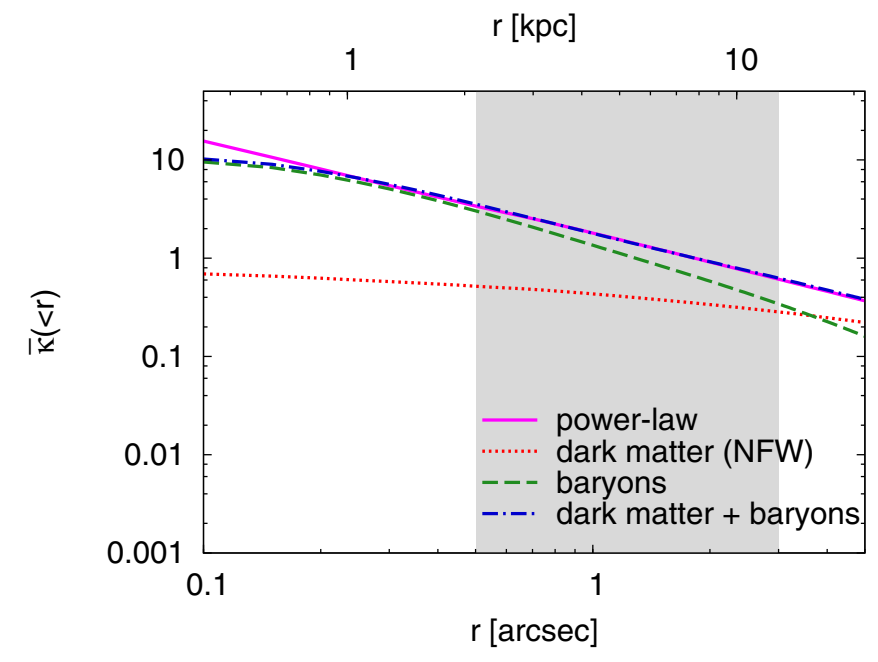

Figure 2. Circularly averaged convergence as a function of radius for the most probable models. The power-law model (solid) is from SU13. The composite model (dot-dashed) consists of a baryonic mass distribution based on the light profile (dashed), and a dark matter distribution based on an NFW profile (dotted). The convergence includes the contribution from the satellite galaxy. In the region covered by the Einstein ring, between $\sim 0^{\prime \prime} .5$ and $\sim 3^{\prime \prime}$, the slope of the composite model is nearly identical to that of the single power law. The spatially extended Einstein ring covering thousands of intensity pixels provide strong constraints on the local lens mass profile.

(A color version of this figure is available in the online journal.)

We allow the centroid of the NFW halo to vary from the lens galaxy $\mathrm{G}$ with Gaussian uncertainties of $\pm 00^{\prime} \cdot 01$. We adopt a Gaussian prior on $r_{\mathrm{s}}$ of $18^{\prime \prime} .6 \pm 2$ '. 6 based on the weak lensing analysis of the SLACS lenses (Gavazzi et al. 2007) which have similar velocity dispersions to that of RXJ1131-1231. For the other parameters, we impose uniform priors.

We sample the 11 parameters using MCMC for a series of source intensity grids: $50 \times 50,52 \times 52,54 \times 54,56 \times 56,58 \times 58$, $60 \times 60$, and $64 \times 64$. As in SU13, the effects of the source grid resolution dominate the uncertainty on the lens parameters. We conservatively combine the results of the different source resolutions by weighting each equally and approximating the combined PDF with a multivariate Gaussian. In the right panel of Figure 1, we show the reconstructed HST image based on our most probable composite model with $64 \times 64$ source pixels, which reproduces the global features of the observed image. In Figure 2, we show the circularly averaged convergence of the same model. Within the shaded region spanned by the spatially extended arcs, the combination of the baryons (dashed) and the dark matter (dotted) in the composite model yields a nearly perfect power-law profile (dot-dashed). For comparison, the power-law model from SU13 is also plotted in solid. Therefore, the spatially extended arcs and the time delays provide strong constraints on the local profile of the lens mass distribution.

The composite model requires an external shear strength of $\gamma_{\text {ext }}=0.075 \pm 0.005$ at an angle of $80^{\circ} \pm 3^{\circ}$ that is overall consistent with the distribution of external mass concentrations (see Figure 5 of SU13).

\section{IMPACT ON TIME-DELAY DISTANCE}

The cosmological $D_{\Delta t}$ to the lens is affected by the external mass distributions along the line of sight and is related to $D_{\Delta t}^{\text {model by }}$

$$
D_{\Delta t}=\frac{D_{\Delta t}^{\text {model }}}{1-\kappa_{\mathrm{ext}}},
$$

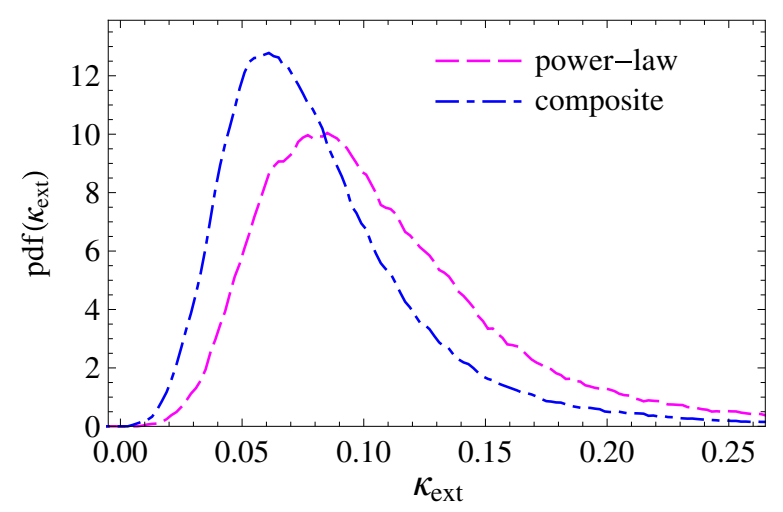

Figure 3. PDF for the external convergence $\kappa_{\text {ext }}$ from combining ray tracing through the Millennium Simulation with (1) the galaxy count around the lens system that is 1.4 times the average number of galaxy counts, and (2) the modeled external shear. The composite model with $\gamma_{\mathrm{ext}}=0.075 \pm 0.005$ is dot-dashed, and the power-law model with $\gamma_{\mathrm{ext}}=0.089 \pm 0.006$ (SU13) is dashed.

(A color version of this figure is available in the online journal.)

where $\kappa_{\text {ext }}$ characterizes the external convergence associated with these mass structures. Following SU13, we construct the PDF of $\kappa_{\text {ext }}$ by ray tracing through the Millennium Simulation (Springel et al. 2005; Hilbert et al. 2009), selecting lines of sight in the simulations with a galaxy count around the lens system that is 1.4 times the average (measured by Fassnacht et al. 2011), and weighting by the external shear value. ${ }^{14}$ In Figure 3 , we show the PDFs of $\kappa_{\text {ext }}$ for the two models, which differ by $\sim 0.02$ due to the shear strengths.

Combining the $D_{\Delta t}^{\text {model }}$ with the $\kappa_{\text {ext }}$ PDF for each of the two lens models, we obtain via Equation (4) the PDF for $D_{\Delta t}$ based on the lensing and time-delay data (Figure 4, left panel). ${ }^{15}$ The $D_{\Delta t}$ PDFs for the two models are shifted with respect to each other by $\sim 4 \%$.

We use the measured lens velocity dispersion of $323 \pm$ $20 \mathrm{~km} \mathrm{~s}^{-1}$ within a rectangular aperture of $0^{\prime \prime} .81 \times 0^{\prime \prime} .7$ (SU13) to further constrain the lens models and help break the mass-sheet degeneracy (e.g., Koopmans 2004). The kinematic modeling of the power-law model is described in SU13. For the composite model, we follow Sonnenfeld et al. (2012) to model the velocity dispersion of the baryonic and the dark matter distributions. In the right panel of Figure 4, we show the resulting $D_{\Delta t}$ PDF by combining lensing, time-delay, and lens-velocity-dispersion measurements. The kinematic data help break lens model degeneracies and provide robust $D_{\Delta t}$ measurements that are less sensitive to lens model assumptions. We conservatively assign equal priors to the two models (power-law and composite), on the grounds that we have no reason to believe one parameterization over another a priori. The combined $D_{\Delta t}$ distribution is shown in solid lines.

A fitting formula for the PDF of $D_{\Delta t}$, which can be used to combine with any other independent data set, is

$$
\begin{aligned}
& P\left(D_{\Delta t} \mid H_{0}, \Omega_{\mathrm{de}}, w, \Omega_{\mathrm{m}}\right) \\
& \quad \simeq \frac{1}{\sqrt{2 \pi}\left(x-\lambda_{\mathrm{D}}\right) \sigma_{\mathrm{D}}} \exp \left[-\frac{\left(\log \left(x-\lambda_{\mathrm{D}}\right)-\mu_{\mathrm{D}}\right)^{2}}{2 \sigma_{\mathrm{D}}^{2}}\right],
\end{aligned}
$$

\footnotetext{
14 Each selected line of sight from the simulation is weighted by the probability of its shear value given the measured value of $0.075 \pm 0.005$ in Section 2.2

15 Assuming a uniform prior on $H_{0}$.
} 

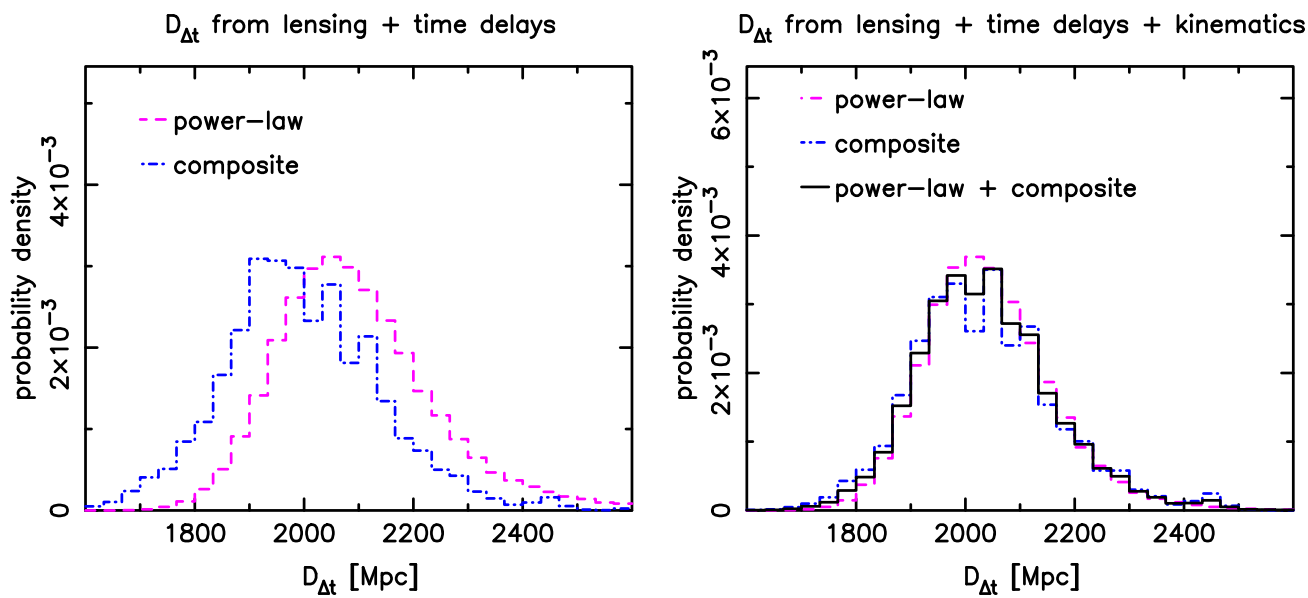

Figure 4. Time-delay distance, $D_{\Delta t}$, for the power-law model (dashed) and the composite model of baryons and dark matter (dot-dashed) in the U $H_{0}$ cosmology. The left panel is based on only the lensing and time-delay data, whereas the right panel includes the information from the lens velocity dispersion. The stellar kinematic information on the lens galaxy helps break lens model degeneracies, yielding very similar $D_{\Delta t}$ distributions for the two lens models. The combined PDF of $D_{\Delta t}$ is shown in solid in the right panel.

(A color version of this figure is available in the online journal.)
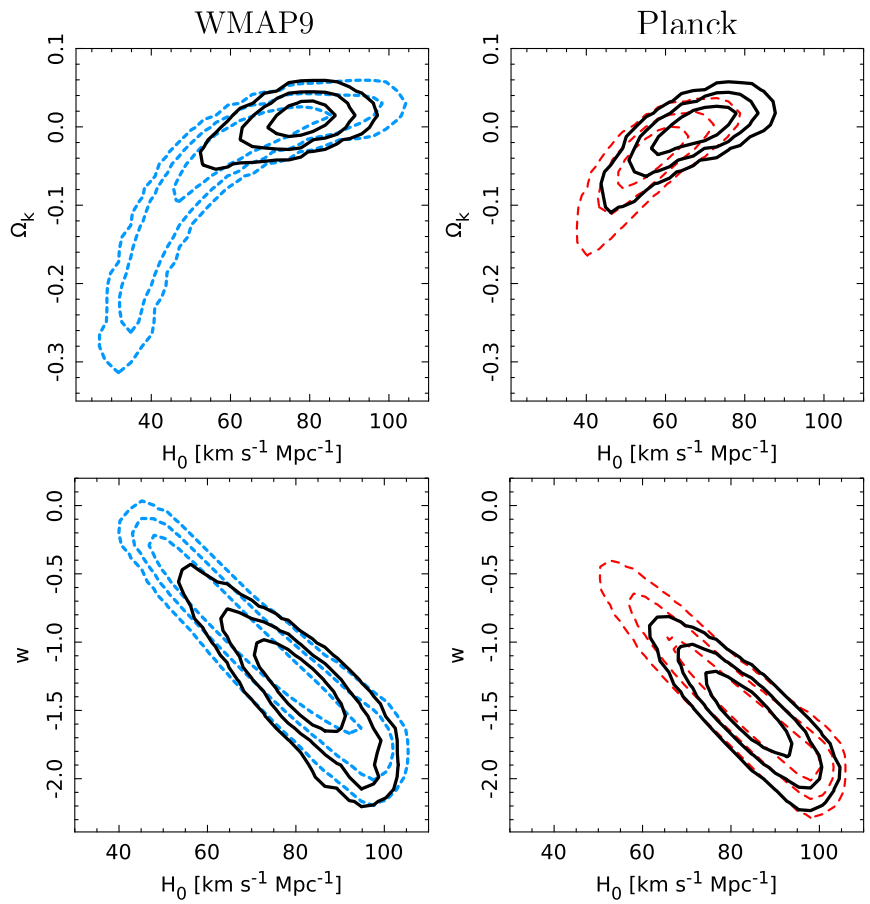

Figure 5. Cosmological constraints assuming open $\Lambda \mathrm{CDM}$ (top) and $w \mathrm{CDM}$ (bottom). Left/Right panels: WMAP9/Planck priors are shown as dashed lines, and the combination of RXJ1131-1231 with WMAP9/Planck is in solid. RXJ1131-1231, which primarily constrains $H_{0}$, helps break parameter degeneracies in the CMB to determine flatness and $w$.

(A color version of this figure is available in the online journal.)

where $x=D_{\Delta t} /(1 \mathrm{Mpc}), \lambda_{\mathrm{D}}=1388.8, \mu_{\mathrm{D}}=6.4682$, and $\sigma_{\mathrm{D}}=0.20560$. Our inference of $D_{\Delta t}$ is accurate to $\sim 6.6 \%$.

\section{COSMOLOGY WITH TIME-DELAY LENSES AND THE CMB}

The time-delay distance allows us to infer cosmological parameters. We consider five background cosmological models, four of which are based on the recent results from WMAP9 (Hinshaw et al. 2013) and Planck (Planck Collaboration et al. 2013): (1) uniform $H_{0}\left(\mathrm{UH}_{0}\right)$ in flat $\Lambda \mathrm{CDM}$ with $\Omega_{\Lambda}=$
$1-\Omega_{m}=0.73$, which is useful for comparing to earlier lensing results, (2) WMAP9 open $\Lambda \mathrm{CDM}$, (3) WMAP9 wCDM, (4) Planck open $\Lambda \mathrm{CDM}$, and (5) Planck $w \mathrm{CDM}$. Compared to the flat $\Lambda \mathrm{CDM}$ model, open $\Lambda \mathrm{CDM}$ allows for spatial curvature $\Omega_{\mathrm{k}}$, and $w \mathrm{CDM}$ allows for a time-independent $w$ that is not fixed to -1 . We consider these more generic models in (2)-(5) given the current tensions in $H_{0}$ measurements from Planck in flat $\Lambda \mathrm{CDM}$ cosmology and from direct probes (Planck Collaboration et al. 2013).

For each of the five cosmological priors, we importance sample the parameters $\left\{H_{0}, \Omega_{\mathrm{m}}, \Omega_{\Lambda}, w\right\}$ from the $\mathrm{U} H_{0}$ prior or WMAP9/Planck MCMC chains ${ }^{16}$ (Lewis \& Bridle 2002; SU13) by weighting with the likelihood of the RXJ1131-1231 data from our improved analysis. In Figure 5, we show the cosmological constraints from the combination of RXJ1131-1231 with WMAP9 (left panels) or with Planck (right panels) in solid contours. Compared to the WMAP9-only or Planck-only constraints (dashed), the gravitational lens RXJ1131-1231 reduces the parameter degeneracies in the CMB data.

The constraint on $H_{0}$ in the $\mathrm{UH}_{0}$ cosmology is $H_{0}=$ $80.0_{-4.7}^{+4.5} \mathrm{~km} \mathrm{~s}^{-1} \mathrm{Mpc}^{-1}$.

The marginalized joint constraints in open $\Lambda \mathrm{CDM}$ are

$$
\left\{\begin{array}{l}
H_{0}=78.0_{-5.1}^{+4.6} \mathrm{~km} \mathrm{~s}^{-1} \mathrm{Mpc}^{-1} \\
\Omega_{\mathrm{k}}=0.011_{-0.007}^{+0.006}
\end{array}\right.
$$

and

$$
\left\{\begin{array}{l}
H_{0}=67.3_{-6.6}^{+6.1} \mathrm{~km} \mathrm{~s}^{-1} \mathrm{Mpc}^{-1} \\
\Omega_{\mathrm{k}}=0.00_{-0.02}^{+0.01}
\end{array} \quad\right. \text { (Planck+RXJ1131). }
$$

The marginalized joint constraints in the flat $w \mathrm{CDM}$ model are

$$
\begin{aligned}
& \left\{\begin{array}{l}
H_{0}=81.4_{-6.2}^{+6.2} \mathrm{~km} \mathrm{~s}^{-1} \mathrm{Mpc}^{-1} \\
w=-1.33_{-0.22}^{+0.20}
\end{array}\right. \\
& \text { and } \\
& \left\{\begin{array}{l}
H_{0}=84.2_{-5.9}^{+6.4} \mathrm{~km} \mathrm{~s}^{-1} \mathrm{Mpc}^{-1} \\
w=-1.52_{-0.20}^{+0.19}
\end{array} \quad\right. \text { (Planck+RXJ1131). }
\end{aligned}
$$

\footnotetext{
${ }^{16}$ For the Planck chains, we use the ones from the Planck temperature power spectrum in combination with WMAP9 low-l polarization data.
} 
The difference in the above marginalized $H_{0}$ with Planck in the two cosmologies is driven by the Planck data. If we restrict $w \geqslant-1$ (the physical regime in most models), then we infer $w=-0.92_{-0.05}^{+0.16}$ with WMAP9 +RXJ1131 and $w=-0.94_{-0.05}^{+0.06}$ with Planck +RXJ1131.

\section{DISCUSSIONS AND CONCLUSION}

The two dominant sources of uncertainty in determining $D_{\Delta t}$ originate from (1) the radial profiles of the lens mass distribution in the region spanned by the images, and (2) weak lensing effects due to mass structures along the line of sight. Recently, SS13 considered toy models of spherical lens mass distributions with point-like sources and suggested that lens models with different radial profiles can lead to $D_{\Delta t}$ values that differ by $\sim 20 \%$ while fitting to the point-like images. We have considered their two lens density profiles in an improved analysis of RXJ1131-1231, and have demonstrated that the spatially extended Einstein ring of the lensed source and the availability of multiple time delays provide strong constraints on the local profile of the lens mass distribution (Figure 2). By incorporating the lens velocity dispersion measurement and estimates of the external convergence $\kappa_{\text {ext }}$, we break degeneracies in the lens model (Figure 4), and show that the results are robust with respect to the chosen form of mass profile at large radii (close to isothermal versus NFW). Work is underway to improve estimates of $\kappa_{\text {ext }}$ (e.g., Greene et al. 2013; Collett et al. 2013; McCully et al. 2014).

By modeling the baryons separately from the dark matter halo, we obtain a rest-frame $M / L_{\mathrm{V}}=7 \pm 3 M_{\odot} / L_{\mathrm{V}, \odot}$ for the baryonic component, where the uncertainty stems mainly from the extrapolation of the lens light profile at large radii. The dark matter mass fraction $\left(f_{\mathrm{DM}}\right)$ within the lens galaxy effective radius of $1^{\prime \prime} .85$ is $\sim 35 \%$. These values are typical of massive early-type galaxies (e.g., Auger et al. 2010; Barnabè et al. 2011). As seen in Figure 2, neither the dark matter nor the baryons is a power law, but the combination of the two leads to a nearly perfect power law locally. This "bulge-halo conspiracy" has already been noted in earlier studies (e.g., Treu \& Koopmans 2004; Koopmans et al. 2009; van de Ven et al. 2009) and is reproduced by some numerical simulations including baryonic physics (Remus et al. 2013).

The centroids of the dark matter halo and the baryonic component of the primary lens galaxy are offset by $\sim 0$ '. 1 , while their position angles agree within $6^{\circ}$. This suggests that the surface mass density of the lens is more complex than a simple elliptical distribution, which is not surprising given the presence of the satellite galaxy. Despite this, the inference of $D_{\Delta t}$ is robust: the various lens model assumptions lead to similar $D_{\Delta t}$, within $\sim 2 \%$, given the exquisite data set. We give a fitting formula in Equation (5) for the PDF of the inferred $D_{\Delta t}$ to RXJ1131-1231 that can be combined with any independent probe of cosmology.

The inferred $H_{0}$ value from Planck in the flat $\Lambda \mathrm{CDM}$ model is $67.3 \pm 1.2 \mathrm{~km} \mathrm{~s}^{-1} \mathrm{Mpc}^{-1}$. This is in tension with several direct $H_{0}$ probes, including the Cepheids distance ladder with $H_{0}=73.8 \pm 2.4 \mathrm{~km} \mathrm{~s}^{-1} \mathrm{Mpc}^{-1}$ (Riess et al. 2011) or $H_{0}=74.3 \pm 1.5$ (stat.) \pm 2.1 (sys.) $\mathrm{km} \mathrm{s}^{-1} \mathrm{Mpc}^{-1}$ (Freedman et al. 2012). Our measurement of $H_{0}$ from RXJ1131-1231 is also in tension with the Planck value under the flat $\Lambda$ CDM assumption. We emphasize that the $H_{0}$ measurements from the CMB are highly model-dependent and can change markedly when one relaxes from spatial flatness or $\Lambda$ (Figure 5). The currently perceived tension could be due to unknown systematic uncertainties or an indication of new physics such as the dark energy component not having $w=-1$. It is now crucial to pin down the uncertainties of each approach and employ multiple independent probes to rule out unknown systematics.

Gravitational lens time delays provide an independent onestep method to determine cosmological distances. With extensive data sets on RXJ1131-1231, we measure its $D_{\Delta t}$ to a precision of $6.6 \%$. We will soon have three more time-delay lenses with similar data quality as that of RXJ1131-1231 and B1608+656 to reduce our statistical uncertainties on cosmological parameters, and more importantly, to test for the presence of residual systematics in our approach. By understanding and eliminating our systematic uncertainties, the statistical power of the hundreds of time-delay lenses from current and upcoming surveys will be realized (e.g., Treu et al. 2013). We are entering an exciting era of accurate cosmology as various methods begin to gain both the precision and accuracy required to rule out cosmological models and potentially discover new physics.

We thank Adriano Agnello, Peter Schneider, Dominique Sluse, Kenneth Wong, and the anonymous referee for useful comments and discussions. We acknowledge support by NASA through HST grant GO-12889. S.H.S. acknowledges support from the Ministry of Science and Technology in Taiwan via grant MOST-103-2112-M-001-003-MY3. T.T. acknowledges support from the Packard Foundation and the National Science Foundation (NSF) grant 1312000. R.D.B. acknowledges support from NSF-AST-0807458. F.C., G.M., and M.T. acknowledge support from the Swiss National Science Foundation. C.D.F. acknowledges support from NSF-AST-0909119. This Letter is based in part on observations made with the NASA/ESA HST, obtained at the Space Telescope Science Institute, which is operated by the Association of Universities for Research in Astronomy, Inc., under NASA contract NAS 5-26555. These observations are associated with program GO-9744.

\section{REFERENCES}

Auger, M. W., Treu, T., Bolton, A. S., et al. 2010, ApJ, 724, 511 Barnabè, M., Czoske, O., Koopmans, L. V. E., Treu, T., \& Bolton, A. S. 2011, MNRAS, 415, 2215

Chávez, R., Terlevich, E., Terlevich, R., et al. 2012, MNRAS, 425, L56 Claeskens, J.-F., Sluse, D., Riaud, P., \& Surdej, J. 2006, A\&A, 451, 865 Collett, T. E., Marshall, P. J., Auger, M. W., et al. 2013, MNRAS, 432, 679 Courbin, F., Chantry, V., Revaz, Y., et al. 2011, A\&A, 536, A53 Dutton, A. A., Brewer, B. J., Marshall, P. J., et al. 2011, MNRAS, 417, 1621 Fassnacht, C. D., Koopmans, L. V. E., \& Wong, K. C. 2011, MNRAS, 410, 2167 Freedman, W. L., Madore, B. F., Scowcroft, V., et al. 2012, ApJ, 758, 24 Gavazzi, R., Treu, T., Rhodes, J. D., et al. 2007, ApJ, 667, 176 Golse, G., \& Kneib, J.-P. 2002, A\&A, 390, 821

Greene, Z. S., Suyu, S. H., Treu, T., et al. 2013, ApJ, 768, 39

Hilbert, S., Hartlap, J., White, S. D. M., \& Schneider, P. 2009, A\&A, 499, 31

Hinshaw, G., Larson, D., Komatsu, E., et al. 2013, ApJS, 208, 19

Humphrey, P. J., \& Buote, D. A. 2010, MNRAS, 403, 2143

Kochanek, C. S., Morgan, N. D., Falco, E. E., et al. 2006, ApJ, 640, 47

Koopmans, L. V. E. 2004, arXiv:astro-ph/0412596

Koopmans, L. V. E., Bolton, A., Treu, T., et al. 2009, ApJL, 703, L51

Koopmans, L. V. E., Treu, T., Bolton, A. S., Burles, S., \& Moustakas, L. A. 2006, ApJ, 649, 599

Lewis, A., \& Bridle, S. 2002, PhRvD, 66, 103511

McCully, C., Keeton, C. R., Wong, K. C., \& Zabludoff, A. I. 2014, arXiv: 1401.0197

Navarro, J. F., Frenk, C. S., \& White, S. D. M. 1996, ApJ, 462, 563

Planck Collaboration, Ade, P. A. R., Aghanim, N., et al. 2013, arXiv:1303.5076

Remus, R.-S., Burkert, A., Dolag, K., et al. 2013, ApJ, 766, 71

Riess, A. G., Macri, L., Casertano, S., et al. 2011, ApJ, 730, 119

Sand, D. J., Treu, T., Ellis, R. S., Smith, G. P., \& Kneib, J.-P. 2008, ApJ, 674, 711

Schneider, P., \& Sluse, D. 2013, A\&A, 559, A37

Sluse, D., Surdej, J., Claeskens, J.-F., et al. 2003, A\&A, 406, L43 
Sonnenfeld, A., Treu, T., Gavazzi, R., et al. 2012, ApJ, 752, 163

Springel, V., White, S. D. M., Jenkins, A., et al. 2005, Natur, 435, 629

Suyu, S. H., Auger, M. W., Hilbert, S., et al. 2013, ApJ, 766, 70

Suyu, S. H., Marshall, P. J., Blandford, R. D., et al. 2009, ApJ, 691, 277

Suyu, S. H., Treu, T., Blandford, R. D., et al. 2012, arXiv:1202.4459
Tewes, M., Courbin, F., \& Meylan, G. 2013a, A\&A, 553, A120

Tewes, M., Courbin, F., Meylan, G., et al. 2013b, A\&A, 556, A22

Treu, T., \& Koopmans, L. V. E. 2004, ApJ, 611, 739

Treu, T., Marshall, P. J., Cyr-Racine, F.-Y., et al. 2013, arXiv:1306.1272

van de Ven, G., Mandelbaum, R., \& Keeton, C. R. 2009, MNRAS, 398, 607 